the sun-spot wave with lowest number (1879-89) with the time of least warmth. If the relative position of all five points does not exactly correspond in the solar and terrestrial curves, we should remember the large uncertainty necessarily attaching to sun-spot measurements.

We might smooth these curves with averages of three, getting the thick line curves, which indicate general decline (in a wide sense), Thus in the case of temperature, we find the grouped sun-spot waves I, 2, 3, associated with more warmth than $2,3,4$, and the latter with more than $3,4,5$.

It is known that the recent researches of Nordmann, extending and confirming the work of Köppen thirty years ago, indicate a state of things in the tropics which is

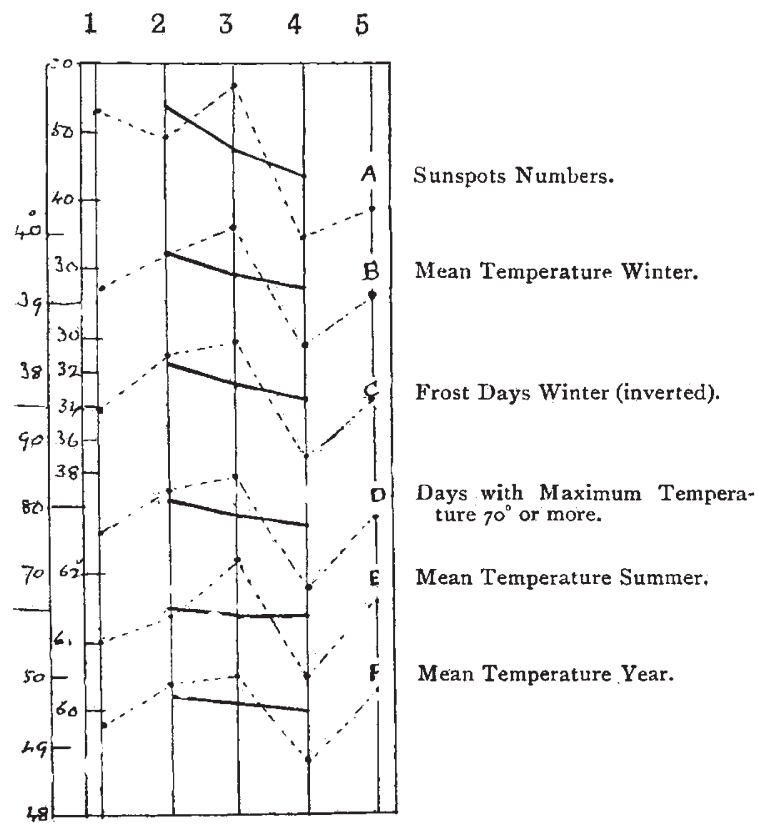

essentially opposite to that in our region, that is, minimum of sun-spots is associated with much heat. (relatively) and maximum with little heat.

I may here be allowed to suomit for criticism a speculation regarding northern regions. I know little of temperature conditions in the Arctic regions and of ice in the Atlantic, and I suppose very little is known of these in their relation to the sun-spot cycle. Let us suppose, however (and the supposition does not seem a wild one), that it is in the Arctic regions as in the tropics, that is, more heat about sun-spot minima than about maxima, and that the Arctic régime is further opposite to ours in showing a general rise of temperature (in a wide sense) since the 'forties. (I may remark that Nordmann's data for the tropics seem to point to a gradual rise in the last thirty years.) What should we expect from this state of things? Would not the greater heat with minima cause more melting and loosening of ice, a more open season, and more ice to be carried down into the Atlantic? This would have a cooling effect on the Gulf Stream, and our temperatures would correspondingly decline. Thus heat in the Arctic would mean cold to us, and a gradual rise of temperature in the far north would mean a gradual fall in western Europe.

I have been told that a certain shrinking of the northern ice covering has been noticed in places in recent times, and I read lately, in connection with the voyage of the Discovery, that since Ross's time the Antarctic ice pack has broken back some thirty miles.

There may, however, be facts adverse to the above theory. Perhaps some of your readers may be able to throw light on the subject. Alex. R. MacDowall.

\section{A NEW EPOCH IN SOLAR PHYSICS.}

$U^{\mathrm{P}}$ to the year 1868 those rose coloured appendages round the solar limb, the prominences, could only be observed at the times of total eclipses of the sun. The ingenious method for watching these phenomena any time when the sun shines we owe to the labours of Lockyer and Janssen, and the striking of a medal by the French Government in honour of this important solar physical advance properly noted an epoch in this branch of astronomy.

By this new device, which was spectroscopic, the positions, forms, structure and movements of the prominences that encircle the solar disc could be accurately watched and determined, and we owe a debt of gratitude to such men as Respighi, Tacchini, Ricco, Mascari and others for the great work they have accomplished in taking advantage of this new line of research by recording daily the state of the solar limb in respect to these appendages. It must not be forgotten that all this work has been accomplished by eye observations alone. Sweeping round the solar limb and noting accurately the position, form, $\& c$. , of each prominence is not the work of a moment, even if the sky is clear, and it is astonishing what a great amount of valuable information has been gathered by this apparently sluggish method. When it is considered that one sweep of the spectroscopic slit round the solar limb only makes us acquainted with the prominences that exist in a very small section of the solar atmosphere and at only one particular moment of time, it was natural that early attempts were made not only to employ photography as a means of quickly recording these, but of devising, if possible, some method by which prominences on the solar disc itself could be also photographed.

It is not the object of the present article to trace the history of the development of the instrument, the photospectroheliograph, which now affords a means of satisfying these and other unlooked for requirements, but to give an account of the latest form adopted and results obtained by Prof. George E. Hale, of the Yerkes Observatory, to whom belongs a large part of the credit of designing and constructing an instrument capable of giving most successful results.

It may nevertheless be mentioned that Janssen in 1869 conceived the first idea of the method; he was followed by Braun, of Kalocsa, in 1872 , and by Lohse, of Potsdam, in I880. In I889 Hale commenced work in this direction, and after him came Evershed in England and Deslandres in Paris, who both designed and used instruments which gave excellent results.

From time to time Prof. Hale has published accounts of the design of, and work accomplished by, his former instruments, but in a recent publication ${ }^{1}$ he gives us a very full and detailed description not only of the latest form he has adopted, but of the magnificent photographs which he has secured with it.

To pass then at once to the modern photospectroheliograph, reference may first be made to the principle involved. The feature of the instrument is that it is capable of giving us pictures of the sun in light of one wave-length, or in monochromatic light. The instrument itself differs little in principle from an ordinary spectroscope if the eye-piece be replaced by a (second) slit. If the solar image be thrown by means of a lens on the first slit, then after the solar light has passed through the lenses and prisms of the spectroscope it will fall on the second slit, which will only allow a narrow portion of the spectrum to pass through it

I Publications of the Yerkes Observatory, vol. iii. part i.; also the Astrophysical Journal. vol. xix., No. I, p. 4I.

NO. I 800, VOL. 69] 
corresponding in width to this slit. The position of this second slit is, however, adjustable, and it can be made to coincide with any line in the solar spectrum. Thus any particular line can be completely isolated by this device. If now the solar image falling on the first slit be kept stationary, and the whole spectroscope be moved in a plane at right angles to the axis of the incident solar beam and in a direction at right angles to the length of the first slit, then the light that will pass through the second slit will be a succession of images in monochromatic light corresponding to the strips of the solar image which entered the first slit.

By adjusting the second slit on, say, the $K$ line of calcium, and placing a photographic sensitive plat $\epsilon$ fixed independently of but nearly in contact with the surface of the second slit, then while the first slit is passing over the solar image, the second is moving over the film of the photographic plate at rest and allowing the monochromatic calcium $\mathrm{K}$ light to impress its successive images on the plate. The result is a picture of the sun in " $\mathrm{K}$ " light. Isolating different lines by means of the second slit, various monochromatic images of the sun in different "lights " can be secured.

An instrument made on the above principle is the ideal form to be used, but it can only be adopted when sunlight is thrown by means of a heliostat on to the lens which forms the image. It may be mentioned, by the way, that an instrument on this principle has recently been erected at the Solar Physics Observatory, South Kensington.

Prof. Hale wished, however, to employ his spectroheliograph in connection with the great Yerkes refractor of 40 inches aperture, so that he was obliged to adopt another method, because the movement of the spectroheliograph, which is of considerable weight, across the solar image formed at the focus of this large lens would have made the telescope vibrate, and produced in consequence bad solar images.

The method which he eventually adopted was to keep the whole spectroheliograph still in relation to the telescope itself, and to move the solar image uniformly across the first slit by means of a motor which actuated the declination slow motion of the telescope. Another difficulty then arose as regards the movement of the photographic holder, for this had to be made to travel across the second slit at the same uniform speed as the image over the first slit; this was finally overcome by connecting the plate holder directly with the declination motor, thus moving them simultaneously.

Although very similar in principle to the ordinary spectroscope, the spectroheliograph is different from it in many important details. In the first place both slits, instead of being straight as is usually the case in spectroscopes, are curved, and curved to a radius which is determined by the material of the prisms employed. Again, it is most convenient if the two tubes, carrying each a slit with its respective objective, and corresponding to the collimator and telescope tubes of the ordinary spectroscope, are arranged parallel to each other. This is accomplished by inserting between the collimator lens and the prisms (two in this case) a plane reflector which can be so adjusted that the light, after being reflected and passing through the prisms, emerges parallel to the beam falling on the reflector or to the collimator's axis.

By the use of the reflector in this position, thus rendering the prisms clear of the optical axis of the collimator, the instrument may be employed for another line of solar research, because when greater dispersion is required, as will be described further on, a grating may be inserted in its place. The optical arrangement, as briefly described above, can be seen from the accompanying illustration (Fig I), which No. I 800 , VOL. 69] shows this portion of the instrument alone. To indicate the path of the beam of light from the collimator objective to the second slit objective a white line has been drawn, the direction being indicated by the arrow head.

For further information regarding the details of the construction of the instrument the reader may be referred to Prof. Hale's account, but a few dimensions may be given here. Since the solar image formed by the forty-inch telescope measures seven inches in diameter, the two slits, both being supplied with the necessary means of adjustment, are eight inches long. The collimator and camera lenses are of the portrait type by Voigtländer, and are of equal aperture $\left(6 \frac{1}{4}\right.$ inches) and focal length. 'These lenses are really too small for the large solar image dealt with, but Prof. Hale's statement that the considerable cost of lenses of about ten inches, the required size, rendered their purchase difficult explains this defect.

Passing over several important points that are vital to the efficient working of this instrument, which would

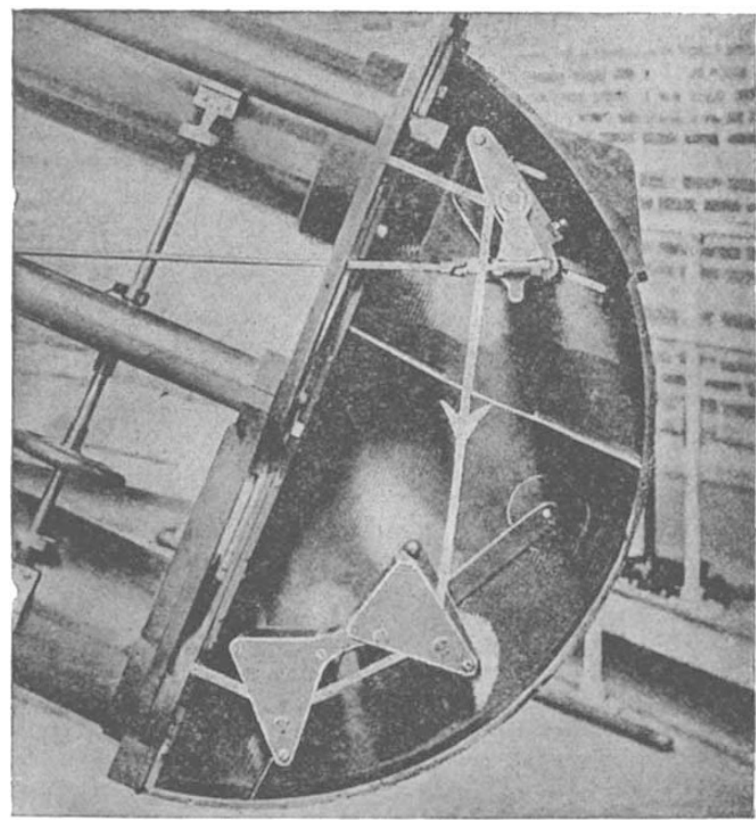

Fig. 1.- Showing the optical arrangement of the Spectroheliogxaph.

here take up too much space even to refer to at short length, such as a description of the movable plateholder, the adjustment of the several parts of the spectroheliograph, the value of diaphragms to reduce diffuse and reflected light, the method of setting the second slit on any particular line in the spectrum, \&c., we now come to describe some of the results which are the first fruits of this research.

It has been stated above that if a line due to calcium be isolated by the second slit then we shall obtain a picture of the sun in calcium light; if a hydrogen or iron line be used, then a hydrogen or iron solar picture will be obtained. The lines which are the most easy to employ, and which give the best results, are those of $H$ and $K$ due to calcium. These lines in the solar spectrum (Fig. 2) are broad, of a composite structure, and are composed of three main parts, (I) a broad, dark band, designated by Prof. Hale as $\mathrm{H}_{1}$ or $\mathrm{K}_{1}$, (2) a comparatively narrow, bright line, lying at the centre of this band at points on the sun's disc where the slit crosses hot masses of calcium vapour $\left(H_{2}, K_{2}\right)$, 
and $(3)$ a very narrow, dark line running through the centre of $\mathrm{H}_{2}$ and $\mathrm{K}_{2}$ designated $\mathrm{H}_{3}$ and $\mathrm{K}_{3}$.

Now according as the second slit is made to isolatt any part of either of these lines, so a calcium picture of the sun corresponding to this particular part of the line is obtained.

It may perhaps be mentioned that the name "flocculi" is here employed to designate the clouds of vapour which are photographed with the spectroheliograph. When the calcium line is employed "calcium flocculi" are photographed, or if the hydrogen line be used we obtain " hydrogen flocculi." These flocculi are, according to Prof. Hale, situated at a greater level above the photosphere than the "faculæe," the latter being elevated regions of the photosphere.

As the width of the $\mathrm{H}$ and $\mathrm{K}$ bands depends on the density of the calcium vapour, and the denser calcium in the sun is below that of less density, the pictures of the sun secured by using different parts of these lines will correspond to different levels. In fact, a means is thus employed of photographing sections of the calcium vapour, or of sounding the solar atmosphere with respect to this element. Thus, if the second

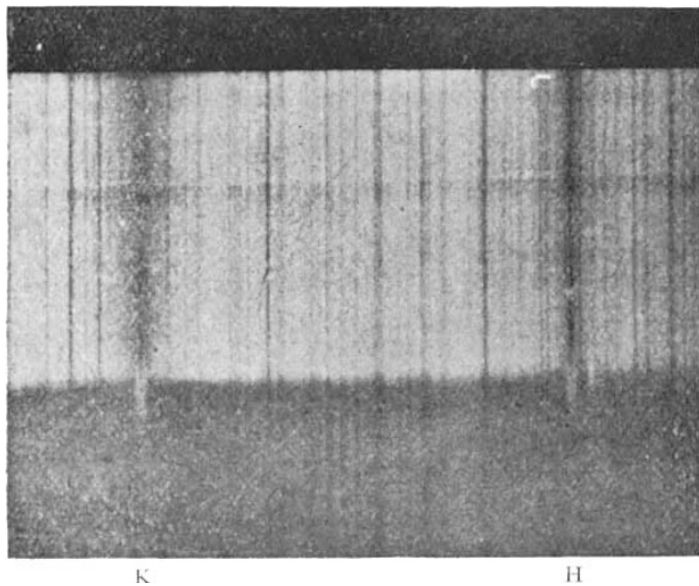

EIG. 2.-Reproduction of a photograph showing the $\mathrm{H}$ and $\mathrm{K}$ lines of calcium in the solar spectrum when large dispersion is employed.

slit be set at the extreme edge of $\mathrm{H}_{1}$ or $\mathrm{K}_{1}$, the resulting photograph will only show that calcium vapour which is dense enough to produce a line of this breadth, in fact, a section across the base of the calcium flocculus will be obtained. If set nearer the centre of the line a section of the flocculus corresponding to a higher level will be produced.

Further, this calcium picture in the final positive is always bright on a dark background no matter which parts of the $\mathrm{H}$ or $\mathrm{K}$ lines are employed. That this is so can be seen from Prof. Hale's pictures taken in the $\mathrm{H}_{1}$ or $\mathrm{H}_{2}$, or $\mathrm{K}_{1}$ or $\mathrm{K}_{2}$ light; this is an important point to which reference will be made later.

An examination of several photographs has led Hale to deduce that the calcium fiocculi when secured with the slit nearer $\mathrm{H}_{3}$ or $\mathrm{H}_{2}$, or $\mathrm{K}_{3}$ or $\mathrm{K}_{2}$ are more extensive than those taken near the outer edge of $\mathrm{H}_{1}$ or $\mathrm{K}_{1}$. It is argued from this that the calcium flocculi are most probably in general composed of a series of columns of vapour expanding as higher levels are reached, and possibly overhanging laterally (Fig. 3).

To explain this point a little more fully perhaps the accompanying diagram (Fig. 4) may prove of service. In the centre of the diagram is drawn (on rather an exaggerated scale) the $\mathrm{H}$ line of calcium, and below NO. I 800 , vOL. 69] this are lettered the detailed portions of it according to Hale's nomenclature. Above this are drawn three layers to represent three strata of calcium vapour corresponding to the width of the $\mathrm{H}$ line, which varies according to the density of the vapour. To investigate the distribution of the densest layer the portion $H_{1}$ of the $\mathrm{H}$ line is used, $\mathrm{H}_{2}$ is employed for the less dense layer, and $\mathrm{H}_{3}$ for the least dense.
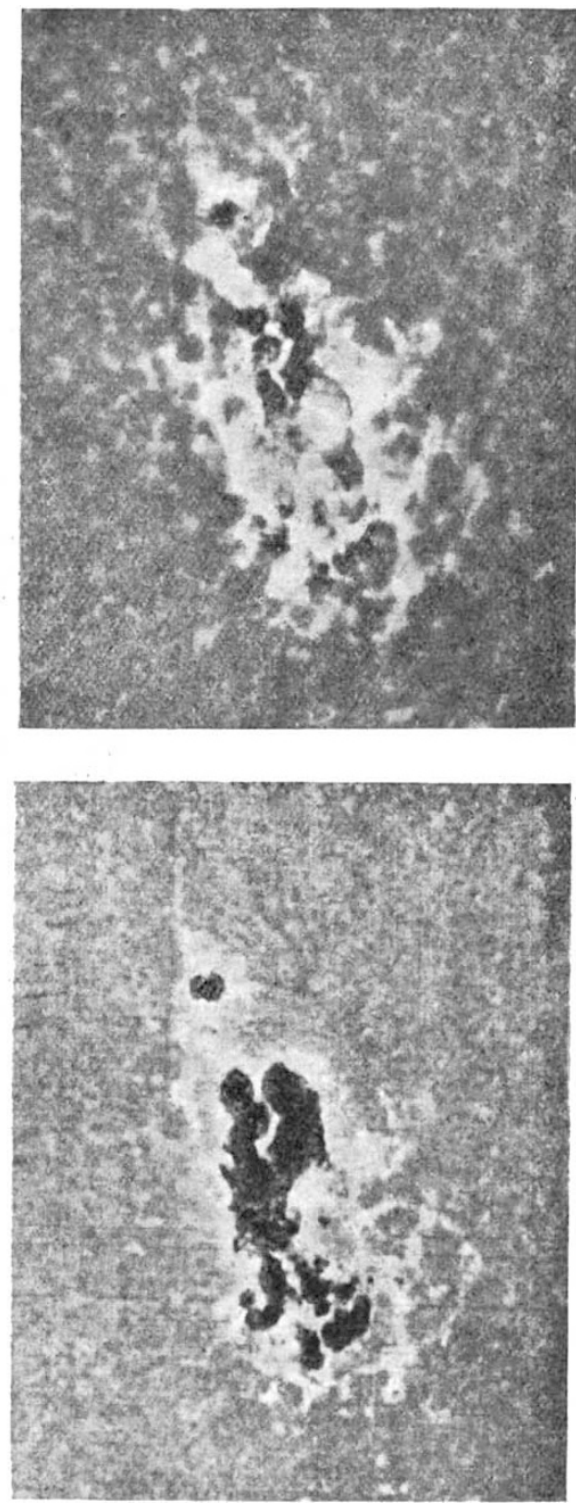

Fig. 3.- -Showing that the bright calcium flocculi are more extensive at the $\mathrm{H}_{2}$ level (upper photograph) than at the $\mathrm{H}_{1}$ level (lower photograph). Notice that the spot is nearly obliterated at the $\mathrm{H}_{2}$ level.

At the right-hand side of Fig. 4 is a sketch of a portion of the solar surface taken separately with $H_{1}$, $\mathrm{H}_{2}$, and $\mathrm{H}_{3}$ lines, and we have therefore the $\mathrm{H}_{1}, \mathrm{H}_{2}$, and $\mathrm{H}_{3}$ flocculi. As, according to Hale, the area of the $\mathrm{H}_{3}$ flocculus is more extensive than that of $\mathrm{H}_{2}$, and $\mathrm{H}_{2}$ more extensive than that of $\mathrm{H}_{1}$, if we project this to gain a mental image of the vertical distribution of this calcium vapour we obtain an object somewhat after that drawn above the three calcium flocculi. 
In fact, the object will take a form which is very like a tree-like prominence.

It is therefore of great interest to examine the same region of a large spot as photographed at two different levels, as in Fig. 3.

This illustration shows the region of the large spot of October 9 of last year, and the. secondary, slit was

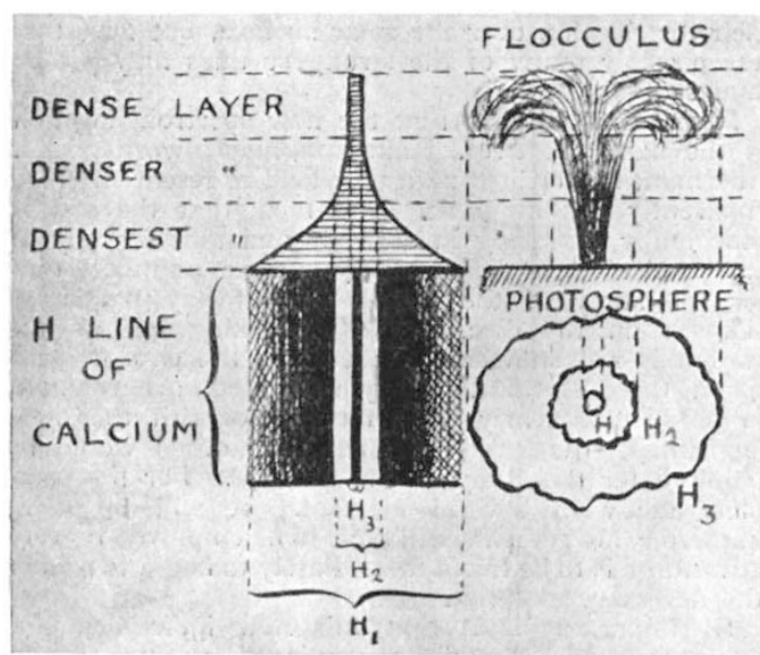

F1G. 4 -Diagram to illustrate the relation between the various portions of the $H$ calcium line, the layers of different density, the resulting extent of the calcium físculi and the probable appearance of their vertical sections

placed at the middle of the calcium $\mathrm{H}_{1}$ level (lower picture), and at the calcium $\mathrm{H}_{2}$ level (upper picture); the plates were exposed at $3 \mathrm{~h} .43 \mathrm{~m}$. and $3 \mathrm{~h} .3 \mathrm{om}$. p.m., so that they may be taken to represent approximately the solar conditions at these two different levels for the same moment of time. The actual spot itself is best seen at the $H_{1}$ level, where the flocculi are not so extensive. At the $\mathrm{H}_{2}$ level the spot is nearly completely covered up by the flocculi, which are here far more pronounced and extensive. At this level the calcium vapour overhangs parts of both the umbra and penumbra of the spot.

The general appearance of the disc of the sun taken in calcium light is shown in the accompanying figure (Fig. 5). Under good atmospheric conditions the whole disc is covered with minute structure resembling somewhat the granulation of the photosphere. Scattered along distinct zones in both hemispheres are large bunches here and there of flocculi.

These masses of flocculi partake of the general movement of rotation of the disc like the spots. Not only do their areas vary very considerably from time to time, but the positions at which they make their first appearance change both as regards latitude and longitude. The enormous extent of these calcium flocculi in relation to the solar disc, and their variation in amount from time to time, a fact also known, suggest that here we have an indication of solar action that has only up to the present time been feebly shown by spots.

It is the investigation of the amount and distribution of these flocculi from day to day and from year to year that makes the spectroheliograph such an important instrument at the present moment, for it is the only means existing of recording these important phenomena.

Not only has Prof. Hale employed the calcium lines in this work, but he has used other lines: motably those of hydrogen. For this investigation the plane reflector used in the optical train of the instrument is replaced by a grating, as larger dispersion of the spectrum has to be obtained. By this means the hydrogen or other lines are rendered somewhat broader, and it then becomes possible to isolate them completely by the second slit, the width of which is adjusted to be less than that of the lines employed, in order to cut out the continuous spectrum on both sides. Numerous photographs, were secured with each of the $\mathrm{H} \beta, \mathrm{H} \gamma$, and $\mathrm{H} \delta$ lines, and comparisons were made with the calcium photographs.

The striking point which this comparison at once showed was that where on the solar disc the calcium flocculi did not exist the hydrogen flocculi were most apparent (Fig. 6), or, as Prof. Hale says, "the hydrogen flocculi are, in general, dark, and that while they have a general resemblance in form to the bright calcium flocculi, the differences are in many cases very striking."

Perhaps a short digression may here be made, as it does not seem quite clear, at any rate to the writer of this article, what Prof. Hale really means by the terms " dark" hydrogen or "dark" calcium flocculi.

The principle of the spectroheliograph is that if a calcium line be chosen to work with, then the resulting solar disc is built up of two kinds of markings, namely, (a) where the calcium exists (bright), and (b) regions where it does not exist (dark). Further, as has already been pointed out, it does not matter which part of the calcium lines is used, as both the dark and bright parts produce bright calcium flocculi on the completed positive. Again, if a hydrogen line be used we have a disc built up of two kinds of markings, (c) where the hydrogen exists (bright), and $(d)$ where no hydrogen exîsts (dark).

Since the existence of each of these substances on the sun's disc is indicated by bright markings, it is not quite clear why Prof. Hale calls the dark atches

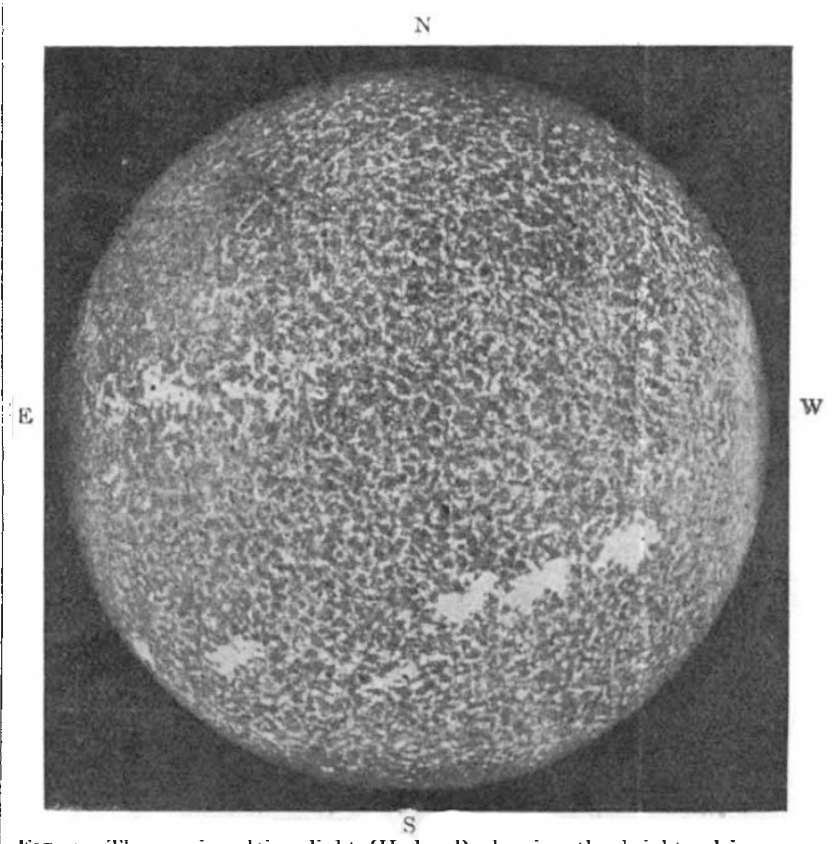

Fig. 5.-The sun in calcium light $\left(\mathrm{H}_{2}\right.$ level), showing the bright ca'cium flocculi, August 12, igo3.

dark calcium or dark hydrogen, as in these parts calcium and hydrogen respectively are, according to the very principle of the spectroheliograph, shown to be absent. NO. I 800, VOL. 69] 
It seems preferable to say that the regions where calcium exists correspond to those regions where hydrogen is absent than to say that the bright calcium flocculi resemble in form the dark hydrogen flocculi.

To show the confusion to which such a form of description as the last mentioned can lead one, a good instance is given on Plate viii., Figs. 3 and 4 , of Prof, Hale's publication. There are shown two illustrations of the same region of the sun, one taken with the calcium line $\left(\mathrm{K}_{2}\right)$, and the other with the hydrogen line $(\mathrm{H} \beta)$. On each of these there is a peculiariy shaped dark patch, evidently the same region on the solar disc, and the photographs show that in this region neither calcium nor hydrogen is present. According to Prof. Hale's notation, this patch should be called both a "dark calcium " and " dark hydrogen flocculus"! As a matter of fact, the marking might be due to quite another substance altogether, and although it appears dark when analysed with either
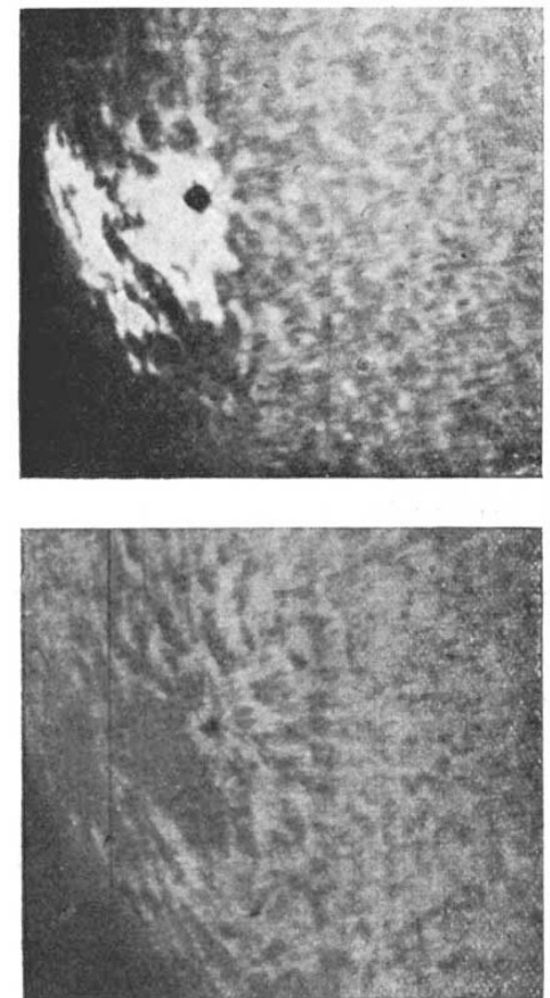

FjG. 6.-Two photographs of the same region of the solar disc taken on the same day, showing that where the bright calcium flocculi are present (upper photograph), the bright hydrogen flocculi (lower photograph) are absent.

the calcium or hydrogen lines, it might appear as a "bright flocculus" if a line in the spectrum of the substance of which it is composed were used. Thus if at the particular levels at which the photographs were secured we knew that helium had been present in this region, then it would have been shown on the photograph as a dark patch if the calcium and hydrogen lines had been employed, and as a bright one if any of the helium lines had been isolated by the secondary slit.

Enough, perhaps, has been said to indicate that what is meant by the "dark hydrogen or calcium" flocculi is not quite clear.

The fact brought out by the beautiful series of photographs of Prof. Hale, that when the bright calcium No. I800, voL. 69] flocculi are absent the bright hydrogen flocculi are present, raises a number of important points in solar physics which the spectroheliograph alone at the present time can attempt to solve.

Calcium and hydrogen are not, however, the only substances which exist in the solar atmosphere. How are the other materials distributed? The comparative thinness of the lines of these other substances in the solar spectrum makes it more difficult to analyse their distribution over the solar surface, but nevertheless possibly many of the strongest lines may yet be analysed.

It will thus be seen that the new spectroheliograph in the hands of Prof. Hale and his co-worker, $\mathrm{Mr}$. Ellerman, has opened up a new field of research which apparently has no limit. The facts that the sun is continually changing in activity and that the sky in any particular place is not always clear point out that for the study of the distribution of any particular element on the disc, one spectroheliograph at one station is not sufficient. Just as in the case of sunspots, three stations, widely separated, are required to produce a nearly daily record, so with this new instrument the same number of stations would be required for the study of one element. For the complete study of several elements, it will be seen, numerous instruments will have to be employed if every advantage is to be taken immediately to begin to gather the necessary material.

So important is it that this new instrument for solar research should be employed to tell us of the changes that are taking place in the sun from day to day and from year to year, that no time should be lost in constructing a sufficient number of them, in distributing them where the raw material, sunlight, can be most often procured, and in organising a homogeneous plan of campaign.

When it is considered that a study of the solar changes is vital for the clear understanding of the numerous terrestrial variations which are so closely associated with our everyday life, the necessity of such a programme is obvious.

Just as in the case of the charting of the heavens, so this work should be of an international character, for every country would be able to reap equally the benefits which such an organisation would bring.

The Rumford spectroheliograph of the Yerkes Observatory having thus shown the exceptional value of this new method of solar research in the hands of Prof. Hale and $\mathrm{Mr}$. Ellerman, future workers will find their task very much lightened by a study of this magnificent and epoch-making contribution to solar physics. It is satisfactory to note that for this work in particular among other valuable contributions to astronomy by the same author, the Royal Astronomical Society has this year awarded Prof. Hale its gold medal. William J. S. Lockyer.

\section{COMMEMORATION DAY AT THE} UNIVERSITY OF GLASGOW.

$\mathrm{N}$ Tuesday, April I9, many of us were thinking of the Kelvin jubilee, which attracted the foremost scientific men of Europe here in 1896 . It is only a fortnight since the body of graduates unanimously elected Lord Kelvin to be our academic chief. In IgoI the university celebrated the ninth jubilee of her own foundation. On April ig she inaugurated an annual commemoration day.

The ceremony opened with a snort religious service. After it Sir William Ramsay, who began his great career as a chemist here, discoursed on Joseph Blackthe most famous chemist who has been connected with 